\title{
Brain Responses to Cardiac Electrical Stimulation: A New EEG Method for Evaluating Cardiac Sensation
}

\author{
Hideaki Suzuki, ${ }^{1}$ Masanori Hirose, ${ }^{1}$ Satoshi Watanabe, ${ }^{2}$ Koji Fukuda, ${ }^{1}$ \\ Shin Fukudo ${ }^{2}$ and Hiroaki Shimokawa ${ }^{1}$ \\ ${ }^{1}$ Department of Cardiovascular Medicine, Tohoku University Graduate School of Medicine, Sendai, Japan \\ ${ }^{2}$ Department of Behavioral Medicine, Tohoku University Graduate School of Medicine, Sendai, Japan
}

\begin{abstract}
Although cardiac sensation, such as palpitation or chest pain, is common and is sometimes a malignant sign of heart diseases, the mechanism by which the human brain responds to afferent signals from the heart remains unclear. In this study, we investigated whether electrical stimulation of the heart provokes brain responses in humans. We examined 15 patients (age: $65.4 \pm 3.1$ years old, 11 males and 4 females) implanted with either a cardiac pacemaker or cardiac resynchronization therapy (CRT) device. Electroencephalogram (EEG) was simultaneously recorded from the vertex during right ventricular pacing at $70-100$ beats/min at baseline $(1.5 \mathrm{~V})$ and intense $(6-8 \mathrm{~V})$ stimulation sessions. We evaluated brain responses to cardiac electrical stimulation by measuring cerebral potentials that were obtained by subtracting the average of 100 EEG waves triggered by cardiac pacing during baseline stimulation from those during the intense stimulation. Intense stimulation of the cardiac pacemaker or CRT device reproducibly induced cardiac sensation in 6 out of the 15 patients; namely, the remaining 9 patients showed no reproducible response. Brain responses were evident by averaging cerebral potentials from all of the 15 patients and those from 6 patients with reproducible cardiac sensation. To the best our knowledge, this is the first report that demonstrates the brain responses to cardiac electrical stimulation in humans. This new method should be useful for examining pathophysiology of cardiac diseases with pathological cardiac sensation, including cardiac anxiety and silent myocardial ischemia.
\end{abstract}

Keywords: cardiac afferent nerves; cardiac resynchronization therapy; cardiac sensation; electroencephalogram; pacemaker

Tohoku J. Exp. Med., 2012, 226 (1), 3-10. C 2012 Tohoku University Medical Press

Cardiac sensation such as palpitation or chest pain is one of the most frequent complaints among patients and sometimes a sign of heart diseases. However, it remains unclear how cardiac sensation is felt. Indeed, sensitivity to cardiac sensation varies among individuals. In general, females and patients with psychiatric disorders are more prone to palpitation and/or chest pain without organic heart disease (cardiac anxiety) (Serlie et al. 1995; Barsky et al. 1996; Ehlers et al. 2000; Barsky 2001). In contrast, arrhythmias are often asymptomatic (Barsky 2001), and painless cardiac ischemic events (silent myocardial ischemia), which are associated with poor prognosis (Canto et al. 2000; Gutterman 2009), are common. Therefore, it is clinically important to elucidate the mechanisms of cardiac sensation in order to improve the quality of life of patients with complaining of cardiac sensation, as well as to improve the prognosis of cardiovascular disease.

Analysis of cerebral evoked potentials (CEPs), obtained by averaging electroencephalogram (EEG) waves triggered by specific events or stimuli, is a standard method to examine brain processing of sensory neuronal activity. Electrical stimulation induces CEPs as well as sensation via activation of somatosensory (Dawson 1947; Lesser et al. 1979) and visceral afferent nerves in various organs, including the esophagus (Frieling et al. 1989a; Hollerbach et al. 1997a, 1997b, 2000; Kanazawa et al. 2000, 2001; Dimcevski et al. 2007), stomach (Dimcevski et al. 2007), duodenum (Dimcevski et al. 2007), rectum (Frieling et al. 1989b; Sinhamahapatra et al. 2001; Watanabe et al. 2007), and bladder (Sarica et al. 1986). We have previously demonstrated that stimulation of the rectum induces brain activation by using CEPs (Watanabe et al. 2007) and brain positron emission tomography (PET) (Suzuki et al. 2009). However, it remains unclear whether CEPs are induced by direct stimulation of the heart.

Cardiac pacemaker and cardiac resynchronization therapy (CRT) device are widely used to treat patients with bradyarrhythmia and heart failure with cardiac dyssyn-

Received October 4, 2011; revision accepted for publication November 13, 2011. doi: 10.1620/tjem.226.3

Correspondence: Hideaki Suzuki, M.D., Department of Cardiovascular Medicine, Tohoku University Graduate School of Medicine, 1-1

Seiryo-machi, Aoba-ku, Sendai 980-8574, Japan.

e-mail: hd.suzuki.1870031@ cardio.med.tohoku.ac.jp 
chrony, respectively, both of which cause electrical stimulation of the heart and thus could potentially induce CEPs. However, there are 3 problems in examining CEPs associated with examining electrical stimulation by a cardiac pacing device. First, from clinical and ethical points of view, it is difficult to stop a cardiac device already in use. Second, since electrical stimulation synchronously contracts the heart, it could also activate afferent receptors outside the heart, such as the great vessel baroreceptors and somatosensory receptors of the chest. Third, the results of heart cyclerelated EEG averaging are substantially contaminated by the cardiac electrical field (Dirlish et al. 1997, 1998; Pollatos and Schandry 2004; Pollatos et al. 2005) (Fig. 1). Despite these problems, cerebral responses to stimulation of the heart with continuous cardiac pacing can be assessed by increasing the stimulation intensity, which augments CEP amplitudes (Lesser et al. 1979; Hollerbach et al. 1997a).

In this study, we aimed to develop a new method for examining brain activation in response to cardiac electrical stimulation by increasing the stimulation intensity of cardiac pacemaker or CRT device.

\section{Materials and Methods}

\section{Patients}

We recorded EEGs from 15 patients (age: $65.4 \pm 3.1$ years old; 11 males, 4 females) with cardiac pacemakers $(n=13)$ or CRT devices $(n=2)$ (Medtronic Inc. in 11 and Lifeline Inc. in 4) (Table 1). All patients provided their written informed consents for their participation. This study was approved by the Ethics Committee of Tohoku University School of Medicine (\#2006-67).

Study protocol (Fig. 2)

All studies were performed during patient hospitalization (4.6 \pm 0.6 days after the device implantation). For all patients, the device setting was changed before examination to baseline stimulation in VVI mode (Inter-Society Commission for Heart Disease Resource) with an intensity of $1.5 \mathrm{~V}$ at $70-100(80.0 \pm 2.6)$ beats/min to maintain right ventricular (RV) pacing throughout the study. Examination was conducted with the patient in the spine position in the EEG room of our hospital. EEGs and ECGs were recorded for 2 min during the baseline stimulation $(1.5 \mathrm{~V})$ and during the intense stimulation (6-8 V) session, which occurred $30 \mathrm{~min}$ after stabilization of the baseline stimulation. Once EEG and ECG recordings of the intense stimulation had been performed, the stimulation intensity was returned to that of the baseline stimulation. EEGs and ECGs were re-recorded during a $2^{\text {nd }}$ session with the baseline and intense stimulation separated with a 10-min interval. All the participants were asked about any chest symptoms that occurred during the sessions by the same investigator after each EEG and ECG recording session. After completion of the study, the device settings were returned to their original state.

\section{EEG and ECG recordings}

An EEG electrode was placed on the vertex $(\mathrm{Cz}$, international 10-20 system) and ECG electrodes were placed on the midline of the right clavicle and the midline of the left $6^{\text {th }}$ intercostal space. An electrode was also placed on the tip of the nose. EEGs and ECGs were recorded with a digital EEG recorder (EEG-1714 Neurofax,
Nihon Kohden, Tokyo, Japan) and were digitized at a sampling rate of 1,000 Hz. EEGs and ECGs were band-pass-filtered at $0.01-100 \mathrm{~Hz}$ and $0.01-500 \mathrm{~Hz}$, respectively. All activity was referenced to electrodes of the both ear lobes and grounded with an electrode on the forehead. An electro-oculogram was also recorded with 2 electrodes placed lateral to the outer canthus of each eye. Electrode resistance was maintained below $5 \mathrm{k} \Omega$. Recorded EEGs and ECGs were divided into each epochs lasting $600 \mathrm{~ms}$ each defined as an interval between $100 \mathrm{~ms}$ before and $500 \mathrm{~ms}$ after each ECG spike of pacemaker stimulation that served as a trigger (Vital Tracer, Kissei Comtec, Nagano, Japan). All epochs with an electro-oculogram activity above $50 \mu \mathrm{V}$ were excluded. One hundred epochs from the beginning of recordings were averaged using EP Lyzer II-A, Kissei Comtec. Epochs recorded after the 101 were abandoned. Cerebral responses to cardiac electrical stimulation were obtained by subtracting the average of 100 waves recorded during the baseline stimulation $(1.5 \mathrm{~V})$ from those during the intense stimulation (6-8 V) (Fig. 1).

\section{Cardiac pacemaker and CRT device}

Electrical stimulation of 0.35-0.50 $(0.45 \pm 0.02)$ ms duration was provided by a bipolar electrode that was attached to the right ventricle (RV). Pacing threshold and resistance of the RV were 0.45 $\pm 0.02 \mathrm{~V}$ and $1001 \pm 90 \Omega$, respectively.

\section{Statistical analysis}

All results were analyzed by the Student's $t$-test or Fisher's exact test and are expressed as mean \pm SEM. A value of $P<0.05$ was considered to be statistically significant.

\section{Results}

Cardiac sensation induced by cardiac electrical stimulation

Cardiac electrical stimulation repeatedly induced cardiac sensation in 6 of the 15 patients. In the remaining 9 patients, 3 subjects showed inconsistent sensation and 6 did not feel any sensation (Table 2). All patients reported the sensation as tolerable and painless. Although the number of patients was small $(n=15)$, patients with reproducible cardiac sensation tended to be younger than those without it $(59.8 \pm 5.6$ vs. $71.5 \pm 1.8$ years, $P=0.076)$ and all 4 female and only 2 of the 11 male subjects had cardiac sensation (Tables 1 and 2).

\section{CEPs induced by cardiac electrical stimulation}

The averaged 100 epochs were contaminated with cardiac electrical field at $\mathrm{Cz}$ and at the tip of the nose during both the baseline and intense stimulation sessions (Fig. 1). Since the shapes of the cardiac electrical fields were similar in the baseline and intense stimulation, pure EEG components associated with cardiac electrical stimulation were obtained by subtracting the average of 100 epochs recorded during the baseline stimulation from those recorded during the intense stimulation (Fig. 2). CEPs were reproducibly noted at $\mathrm{Cz}$ in the averaged waves of all 15 patients (the first negative peak (N1), 65/64 msec; the first positive peak (P1), 127/141 msec; the second negative peak (N2), $166 / 213$ msec; the peak-to-trough amplitude of the N1 and P1 waves (N1/P1), 1.67/1.19 $\mu \mathrm{V}$ and the peak-to-trough 


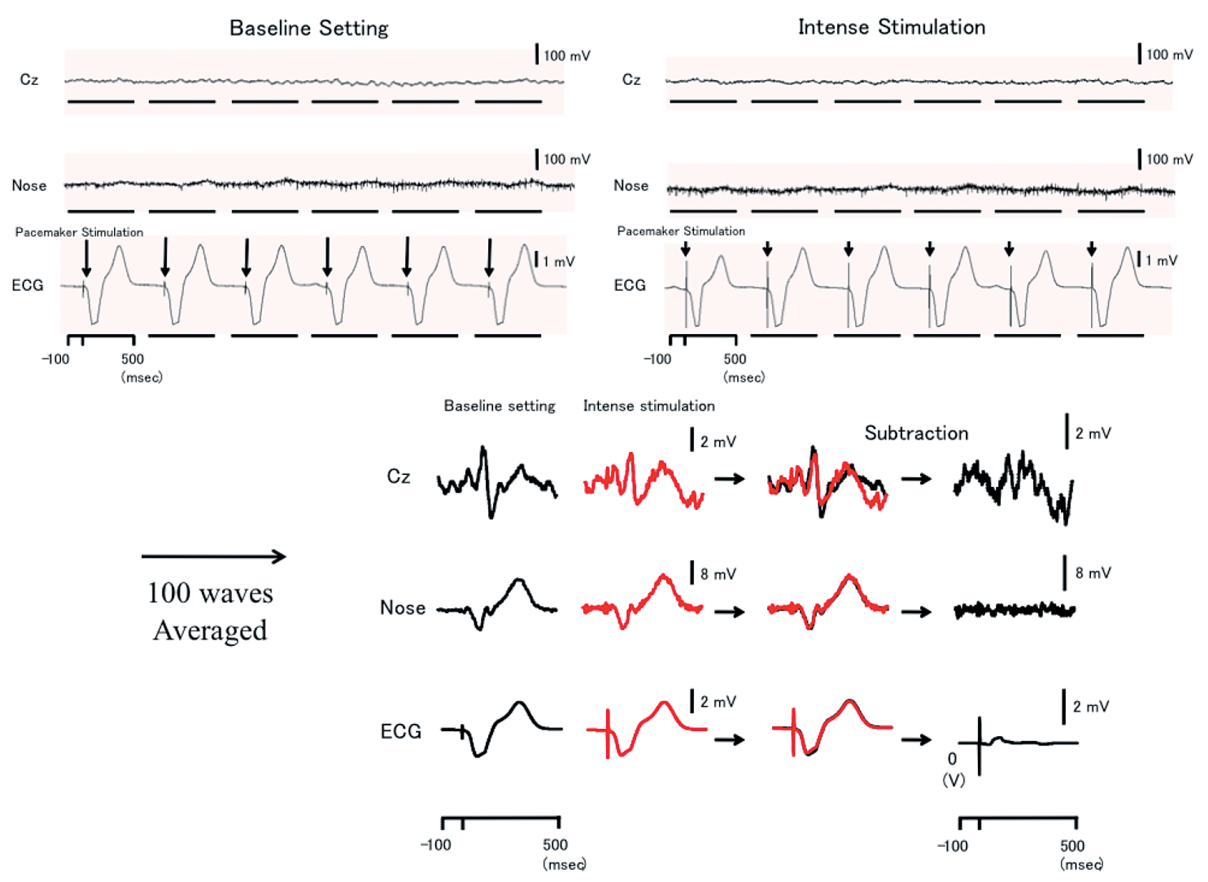

Fig. 1. Measurement of CEPs in response to cardiac electrical stimulation.

EEG and ECG recordings of a patient (No.8) during the $1^{\text {st }}$ session. Electrical waves at the vertex $(\mathrm{Cz})$ contain both EEG and ECG components, while those at the nose and chest contain only ECG components. Since the ECG components were not significantly different between the baseline and intense stimulation sessions, pure EEG components (CEPs) at $\mathrm{Cz}$ were obtained by subtracting averaged waves recorded during the baseline stimulation from those recorded during the intense stimulation. CEPs, cerebral evoked potentials; ECG, electrocardiogram; EEG, electroencephalogram.

Table 1. Patient characteristics.

\begin{tabular}{lccccccccccl}
\hline & Diagnosis Pacemaker & Age & Sex & SBP & DBP & HR & DM & HF & NYHA & History \\
\hline No. 1 & SSS & VVI & 74 & Male & 104 & 65 & 60 & + & + & 2 & chronic Af \\
No. 2 & AVB & DDD & 66 & Female & 140 & 60 & 61 & - & - & 1 & no history \\
No. 3 & AVB & DDD & 62 & Female & 104 & 69 & 72 & + & - & 1 & sarcoidosis \\
No. 4 & AVB & VVI & 70 & Male & 120 & 60 & 50 & - & + & 2 & chronic Af \\
No. 5 & SSS & DDD & 79 & Male & 113 & 71 & 70 & - & - & 2 & aortic stenosis, paroxysmal Af \\
No. 6 & AVB & DDD & 56 & Male & 99 & 58 & 62 & + & - & 1 & dilated cardiomyopathy \\
No. 7 & AVB & DDD & 66 & Male & 120 & 60 & 60 & - & - & 1 & no history \\
No. 8 & SSS & DDD & 37 & Male & 129 & 72 & 55 & - & - & 1 & sarcoidosis \\
No. 9 & SSS & DDD & 70 & Male & 165 & 88 & 60 & - & - & 1 & abdominal aortic aneurythm, paroxysmal Af \\
No. 10 & SSS & DDD & 70 & Male & 103 & 65 & 60 & - & - & 1 & no history \\
No. 11 & AVB & DDD & 59 & Female & 134 & 87 & 53 & - & - & 1 & no history \\
No. 12 & AVB & CRT & 79 & Female & 85 & 38 & 72 & - & + & 2 & dilated cardiomyopathy \\
No. 13 & SSS & DDD & 77 & Male & 122 & 68 & 52 & - & - & 1 & no history \\
No. 14 & CLBBB & CRT & 71 & Male & 114 & 77 & 93 & + & + & 1 & myocardial infarction, chronic Af \\
No. 15 & SSS & DDD & 45 & Male & 125 & 77 & 60 & - & + & 2 & aortic \& mitral valve replacement, chronic Af \\
\hline
\end{tabular}

History of DM and HF expressed as with $(+)$ or without $(-)$.

VVI and DDD are quoted from the Inter-Society Commission for Heart Disease Resource. Af, atrial fibrillation; AVB, advanced ventricular block; CLBBB, complete left bundle branch block; CRT, cardiac resynchronized therapy; DBP, diastolic blood pressure; HR, heart rate; SBP, systolic blood pressure; SSS, sick sinus syndrome; DM, diabetes mellitus; HF, heart failure.

amplitude of the P1 and $\mathrm{N} 2$ waves (P1/N2), 1.45/0.99 $\mu \mathrm{V}$ expressed as the results of the $1^{\text {st }}$ and $2^{\text {nd }}$ session (Fig. 3). The shape of the CEPs was more evident in the averaged waves of the 6 patients who repeatedly felt cardiac sensation $(\mathrm{N} 1,66 / 114 \mathrm{msec} ; \mathrm{P} 1,126 / 169 \mathrm{msec} ; \mathrm{N} 2,183 / 186$ msec; N1/P1, 2.98/1.77 $\mu \mathrm{V}$ and $\mathrm{P} 1 / \mathrm{N} 2,2.49 / 1.84 \mu \mathrm{V}$ 
Table 2. Cardiac sensation in response to cardiac electrical stimulation.

\begin{tabular}{|c|c|c|}
\hline Patient & 1st time & 2nd time \\
\hline No. 1 & - & - \\
\hline No. 2 & anything uncomfortable in the chest & a little uncomfortable \\
\hline No. 3 & itching at the apex & beating on the stomach \\
\hline No. 4 & - & - \\
\hline No. 5 & - & - \\
\hline No. 6 & beating lightly on the chest & beating vessels of the whole body \\
\hline No. 7 & - & - \\
\hline No. 8 & heartbeats did not match the heart & palpitation became relieved \\
\hline No. 9 & - & - \\
\hline No. 10 & - & - \\
\hline No. 11 & palpitation became stronger & palpitation became weaker after returning to baseline stimulation \\
\hline No. 12 & palpitation seemed to be stronger & palpitation became stronger \\
\hline No. 13 & seemed to itch at the apex & - \\
\hline No. 14 & felt uncomfortable on the heart & - \\
\hline No. 15 & - & palpitation became stronger \\
\hline
\end{tabular}

Cardiac electrical stimulation repeatedly induced cardiac sensation in 6 patients (Nos. 2, 3, 6, 8, 11, and 12), while other 6 patients (Nos. 1, 4, 5, 7, 9, and 10) had no sensation. Three patients (Nos. 13, 14 and 15) did not show consistent cardiac sensation. “-” represents no sensation.
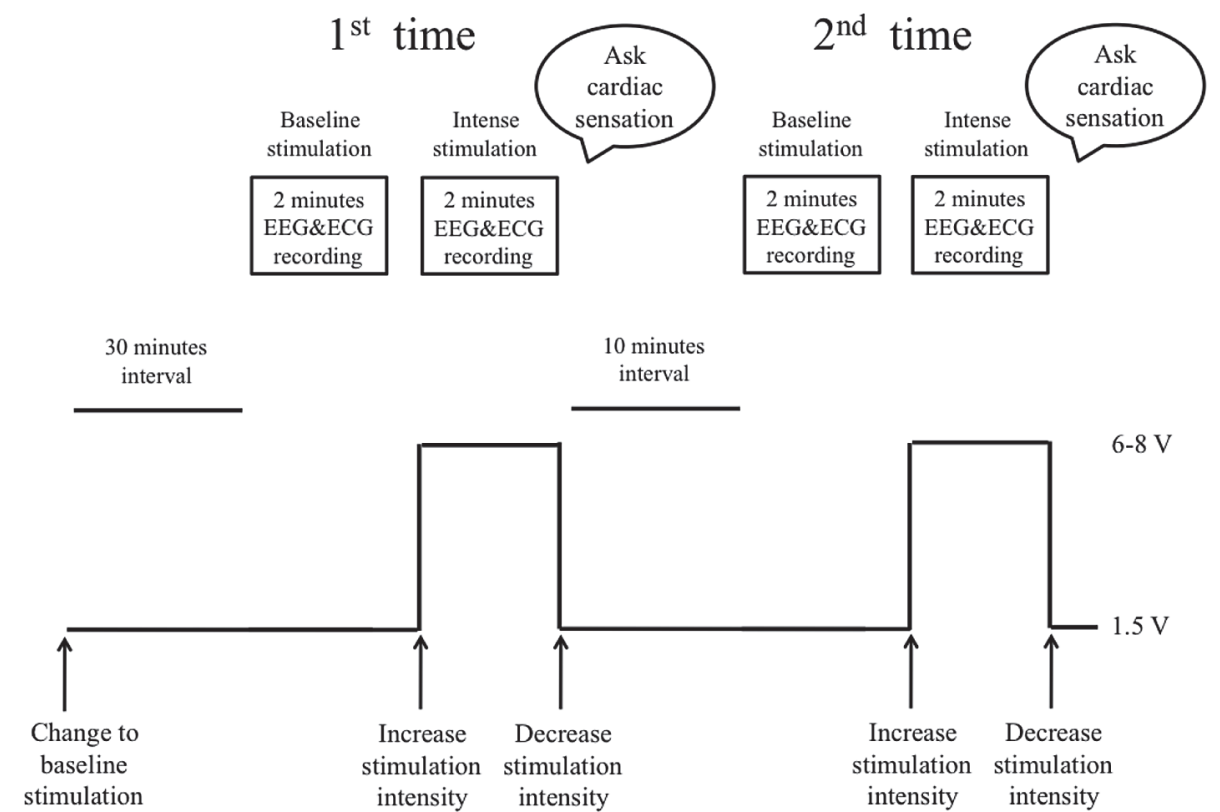

Fig. 2. Study protocol.

The device setting for baseline stimulation was right ventricular pacing of VVI (Inter-Society Commission for Heart Disease Resource) with an intensity of $1.5 \mathrm{~V}$ at 70-100 beats/min. ECG, electrocardiogram; EEG, Electroencephalogram.

expressed as the results of the $1^{\text {st }}$ and $2^{\text {nd }}$ session) compared with the 6 patients with no reported session (Fig. 3).

\section{Discussion}

In this study, we were able to document CEPs in response to cardiac electrical stimulation by increasing in the stimulation intensity of a cardiac pacemaker or CRT device. To the best of our knowledge, this is the first demonstration of CEPs in response to cardiac stimulation. This new method could be useful for examining the interactions between the heart and brain mediated by cardiac afferent nerves.

\section{Validation of the present methods}

Cardiac electrical stimulation can activate not only cardiac afferent nerves but also cardiac contraction and humoral secretion (e.g., brain natriuretic peptide). In this study, the stimulation site was localized at the tip of the 


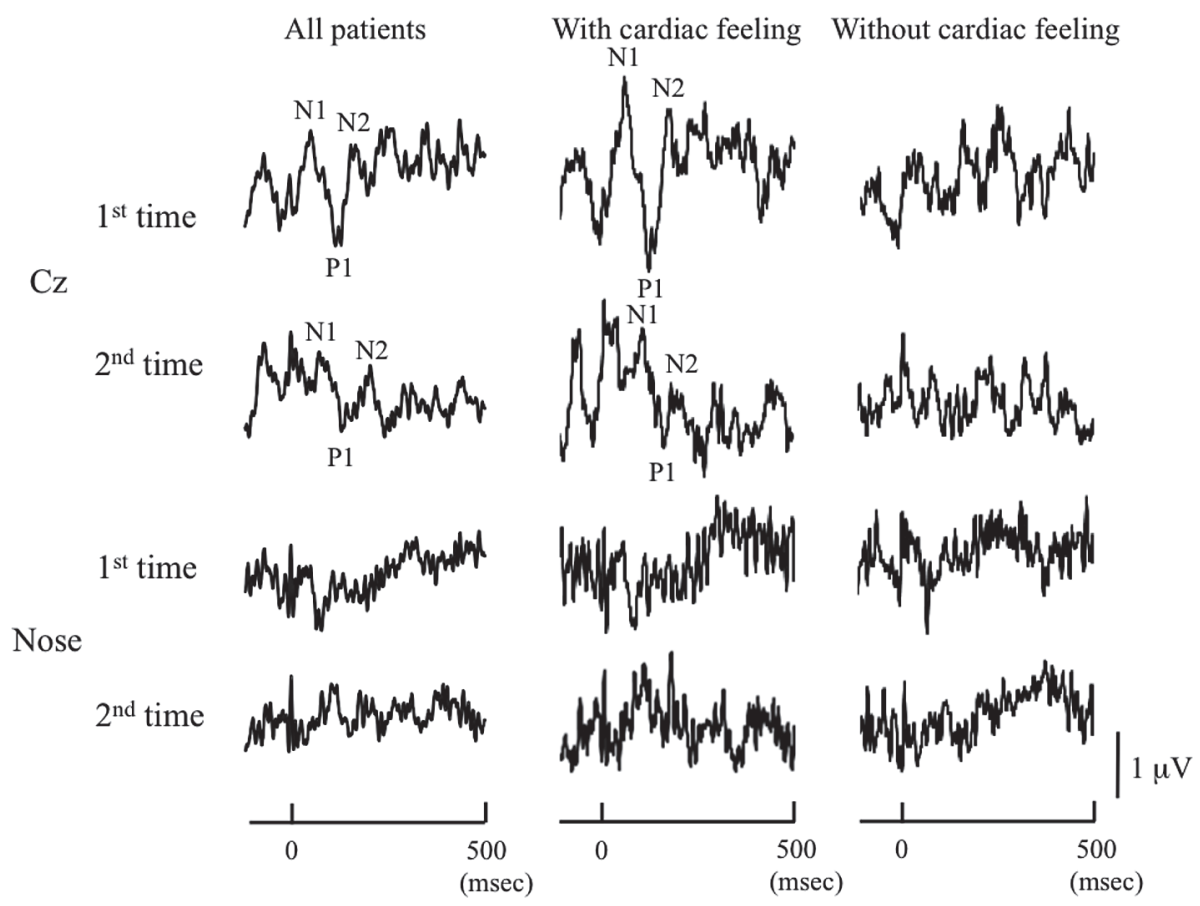

Fig. 3. Cerebral evoked potentials (CEPs) in response to cardiac electrical stimulation.

Electrical waves averaged from all patients $(n=15$, left), patients with reproducible cardiac sensation $(n=6$, center) and patients without it $\left(\mathrm{n}=6\right.$, right) at the vertex $(\mathrm{Cz})$ and the nose. Patients with inconsistent sensation between the $1^{\text {st }}$ and $2^{\text {nd }}$ sessions were excluded $(n=3)$. CEPs were only noted at the $\mathrm{Cz}$ were averaged from all patients as well as from those with cardiac sensation. The peaks of the cerebral responses were designated as the first negative wave (N1), the first positive wave ( $\mathrm{P} 1)$ and the second negative wave $(\mathrm{N} 2)$. The " $0 \mathrm{msec}$ " indicates the stimulus point.

bipolar electrodes and no obvious change in the cardiac electrical field was noted between the baseline and intense stimulation sessions, indicating that the increase in cardiac electrical stimulation intensity caused no significant hemodynamic changes. Cardiac hormones or cytokines may not induce CEPs within a few minutes of cardiac stimulation, although brain natriuretic peptide could inhibit inflammatory pain via nociceptive sensory neurons (Zhang et al. 2010). Thus, CEPs obtained in this study are considered to specifically reflect brain activation mediated by cardiac afferent nerves.

\section{Implications of CEPs with cardiac stimulation}

In this study, the latency of CEPs in response to cardiac stimulation (N1, 64-114 msec; P1, 126-169 msec and $\mathrm{N} 2,166-213 \mathrm{msec}$ ) was comparable with that of CEPs in response to esophageal stimulation $(\mathrm{N} 1,65-121 \mathrm{msec}$; $\mathrm{P}$, 89-194 msec and N2, 128-253 msec) (Frieling et al. 1989a; Hollerbach et al. 1997a, 1997b, 2000; Kanazawa et al. 2000; Dimcevski et al. 2007), which reflect the similar distance between the brain, heart and esophagus. Moreover, similar brain areas may be involved in the sensation of both the heart and esophagus. Patients without organic heart disease occasionally feel angina-like chest pain (non-cardiac chest pain, NCCP) and half of them are subsequently found to have gastrointestinal reflux disease (Bautista et al. 2004). Chest pain induced by gastrointestinal reflux or acid infusion of the esophagus is indistinguishable from angina in patients with symptomatic coronary artery disease (Mellow et al. 1983; Singh et al. 1992). Indeed, a feeling of painless cardiac sensation is mediated by the insula and somatosensory cortex (Critchley et al. 2004; Khalsa et al. 2009), both of which are also activated during esophageal stimulation (Aziz et al. 1997, 2000; Kobayashi et al. 2010). Thus, it is highly possible that CEPs in response to esophageal and cardiac stimulation share the same afferent neuronal pathway to the brain.

CEPs associated with cardiac sensation were first examined as heartbeat evoked potentials (HEPs) which are CEPs triggered by an R wave of the ECG and show a positive potential shift of about $250-600 \mathrm{~ms}$ after the $\mathrm{R}$ wave (Jones et al. 1986; Schandry et al. 1986; Montaya et al. 1993; Dirlish et al. 1997, 1998; Pollatos and Schandry 2004; Pollatos et al. 2005; Gray et al. 2007). HEPs are associated with cardiac sensation as evidenced by the fact that HEP amplitudes are greater in subjects with good heartbeat perception compared with those without it (Pollatos and Schandry 2004; Pollatos et al. 2005). There may be 2 differences between HEPs and CEPs in this study. First, HEPs lack stimulation selectivity to the heart, because heart contractions followed by $\mathrm{R}$ waves activate not only cardiac sensory receptors (Sleight and Widdicombe 1965; Malliani et al. 1973) but also the great vessel baroreceptors (Bronk and Stella 1932; Bloor 1964; Coleridge et al. 1973) and the somatosensory nerves of the chest (Khalsa et al. 2009). Cardiac sensation originates from the heart as well 
as from other organs/tissues (Barsky et al. 1998; Kobayashi et al. 2010). Indeed, one-third of patients with cardiac transplants can perceive their heartbeat correctly (Barsky et al. 1998). Somatosensory receptors of the chest wall are also involved in heartbeat perception (Khalsa et al. 2009). Neurons of the great vessel baroreceptors in the aortic arch and carotid and pulmonary arteries as well as cardiac receptors are activated in a synchronized manner with heartbeat (Bronk and Stella 1932; Bloor 1964; Sleight and Widdicombe 1965; Coleridge et al. 1973; Malliani et al. 1973) and thus could potentially be associated with heartbeat perception. To understand the mechanism of cardiac sensation, it is important to distinguish between cardiac sensation originating from the heart and that originating from the other organs. Second, the positive potential shift of 250-600 msec in HEPs is slower than the latency of the CEPs with cardiac electrical stimulation. Neuronal activation of cardiac receptors and great vessel baroreceptors is followed by R waves with a time delay (Bronk and Stella 1932; Bloor 1964; Sleight and Widdicombe 1965; Coleridge et al. 1973; Malliani et al. 1973). The delay in HEP latency compared with that observed in this study, can be explained by the time gap between ECG R waves and heart cyclerelated neuronal activation that involves cardiac receptors, great vessel baroreceptors, and chest sensory receptors. Thus, CEPs with cardiac electrical stimulation may be more selective to cardiac afferent neuronal activity than HEPs.

\section{Study limitations}

There are several limitations to this study. First, we only enrolled patients with an implanted cardiac device. There is an upper limit of stimulation intensity in cardiac devices (6-8 $\mathrm{V}$ in the pacemakers used in this study). Patients complained of pain in the chest or at the site of pacing at 15-25 mA (Schwedel and Escher 1964). Stimulation of greater intensity could induce cardiac feeling and clear triphasic waves in the patients without cardiac sensation. Electrical stimulation of the heart can potentially be applied to all subjects who can tolerate it using a temporary pacing catheter with greater stimulation intensity than a cardiac device. Thus, the method in the present study should be examined using a temporary pacing catheter. Second, in this study, we only examined cerebral responses to RV stimulation. Patients with anterior or inferior myocardial infarction often present different clinical manifestations (Kapa and Somers 2008). Indeed, neuronal innervation is different among the RV, left ventricles (LV), and both atria (Marron et al. 1994). Thus, cerebral responses evoked by atrial and LV stimulation remain to be examined. Third, the increase in pacing stimulation intensity is only one of the many stimuli of the heart, and the effects of other many stimuli (such as ischemia, changes in contraction, and heart rate) remain to be examined in future studies. Fourth, it has been questioned whether verbal reporting of sensation during visceral stimulation is a reliable and unbiased method for the assessment of visceral sensitivity in man
(Van Oudenhove et al. 2008). In addition, in the present study, all female patients showed reproducible cardiac sensation, suggesting the involvement of psychological factors. Thus, objective markers of visceral sensitivity, such as nociceptive flexion reflex (Sabaté et al. 2008; Van Oudenhove et al. 2008), should be examined in future studies.

Possible application of CEPs in response to cardiac electrical stimulation

The CEPs in the present study can be useful for examining cardiac diseases with pathological cardiac sensation such as cardiac anxiety and/or silent myocardial ischemia. Indeed, CEPs in response to gastrointesitinal stimulation have already been used to examine the pathophysiology of functional gastrointestinal disorders (Hollerbach et al. 2000; Kanazawa et al. 2000, 2011; Sinhamahapatra et al. 2001). Cerebral responses in association with visceral sensation are pathologically altered in functional gastrointestinal disorders such as irritable bowel syndrome (IBS), functional dyspepsia and NCCP (Hollerbach et al. 2000; Kanazawa et al. 2000, 2011; Mertz et al. 2000; Sinhamahapatra et al. 2001; Van Oudenhove et al. 2007, 2008; Watanabe S et al. 2007; Tillisch et al. 2011). They are considered to play an important pathogenic role in functional gastrointestinal disorders (Van Oudenhove et al. 2007). We have previously developed a method for examining CEPs and brain PET during rectal stimulation (Watanabe S et al. 2007; Suzuki et al. 2009) and have suggested that the patients with IBS showed aberrant cerebral responses during rectal stimulation as compared with healthy subjects (Kanazawa et al. 2011). Our finding has been supported by other studies (Silverman et al. 1997; Mertz et al. 2000; Sinhamahapatra et al. 2001; Tillisch et al. 2011).

Since many patients without organic heart disease also complain of cardiac symptoms (Serlie et al. 1995; Barsky et al. 1996; Ehlers et al. 2000; Barsky 2001), there may be some similarities in aberrant cerebral responses between functional cardiac and gastrointestinal disorders. Furthermore, cerebral responses have been shown to be different between patients with angina and those with silent myocardial ischemia during systemic dobutamine infusion (Rosen et al. 1996), although silent myocardial ischemia may be associated with both functional and organic abnormalities (Canto et al. 2000; Gutterman 2009). Therefore, CEPs in response to selective electrical stimulation of the heart could be useful for examining the pathophysiology of cardiac anxiety and/or silent myocardial ischemia.

In conclusion, we were able to develop a new method of recording CEPs in response to cardiac electrical stimulation, which could be useful for examining cardiac sensation in health and disease.

\section{Acknowledgments}

We thank Tomokazu Sasaki and Kenichi Suzuki for their technical assistance with the cardiac pacemakers and CRT 
devices, and Shoko Miura for advice regarding the EEG recordings. This study was supported in part by a grant from the Tohoku University International Advanced Research and Education Organization, Sendai, Japan.

\section{Conflict of Interest}

We have no conflicts of interest to declare.

\section{References}

Aziz, Q., Andersson, J.L., Valind, S., Sundin, A., Hamdy, S., Jones, A.K., Foster, E.R., Långström, B. \& Thompson, D.G. (1997) Identification of human brain loci processing esophageal sensation using positron emission tomography. Gastroenterology, 113, 50-59.

Aziz, Q., Thompson, D.G., Ng, V.W., Hamdy, S., Sarkar, S., Brammer, M.J., Bullmore, E.T., Hobson, A., Tracey, I., Gregory, L., Simmons, A. \& Williams, S.C. (2000) Cortical processing of human somatic and visceral sensation. $J$. Neurosci., 20, 2657-2663.

Barsky, A.J., Delamater, B.A., Clancy, S.A., Antman, E.M. \& Ahern, D.K. (1996) Somatized psychiatric disorder presenting as palpitations. Arch. Intern. Med., 156, 1102-1108.

Barsky, A.J., Ahern, D.K., Brener, J., Surman, O.S., Ring, C. \& Dec, G.W. (1998) Palpitations and cardiac awareness after heart transplantation. Psychosom. Med., 60, 557-562.

Barsky, A.J. (2001) Palpitations, arrhythmias, and awareness of cardiac activity. Ann. Intern. Med., 134, 832-837.

Bautista, J., Fullerton, H., Briseno, M., Cui, H. \& Fass, R. (2004) The effect of an empirical trial of high-dose lansoprazole on symptom response of patients with non-cardiac chest pain-a randomize, double-blind, placebo-controlled, crossover trial. Aliment Pharmacol. Ther., 19, 1123-1130.

Bloor, C.M. (1964) Aortic baroreceptor threshold and sensitivity in rabbits at different ages. J. Physiol., 174, 163-171.

Bronk, D.W. \& Stella, G. (1932) Afferent impulses in the carotid sinus nerve. J. Cell. Comp. Physiol., 1, 113-130.

Canto, J.G., Shlipak, M.G., Rogers, W.J., Malmgren, J.A., Frederick, P.D., Lambrew, C.T., Ornato, J.P., Barron, H.V. \& Kiefe, C.I. (2000) Prevalence, clinical characteristics, and mortality among patients with myocardial infarction presenting without chest pain. JAMA, 283, 3223-3229.

Coleridge, H.M., Coleridge, J.C., Dangel, A., Kidd, C., Luck, J.C. \& Sleight, P. (1973) Impulses in slowly conducting vagal fibres from afferent endings in the veins, atria, and arteries of dogs and cats. Circ. Res., 33, 87-97.

Critchley, H.D., Wiens, S., Rotshtein, P., Ohman, A. \& Dolan, R.J. (2004) Neural systems supporting interoceptive awareness. Nat. Neurosci., 7, 189-195.

Dawson, G.D. (1947) Cerebral responses to electrical stimulation of peripheral nerve in man. J. Neurol. Neurosurg. Psychiatry, 10, 134-140.

Dimcevski, G., Sami, S.A., Funch-Jensen, P., Le Pera, D., Valeriani, M., Arendt-Nielsen, L. \& Drewes, A.M. (2007) Pain in chronic pancreatitis: the role of reorganization in the central nervous system. Gastroenterology, 132, 1546-1556.

Dirlish, G., Dietl, T., Vogl, L. \& Strain, F. (1998) Topography and morphology of heart action-related EEG potentials. Electroencephalogr. Clin. Neurophysiol., 108, 299-305.

Dirlish, G., Vogl, L., Plaschke, M. \& Strain, F. (1997) Cardiac field effects on the EEG. Electroencephalogr. Clin. Neurophysiol., 102, 307-315.

Ehlers, A., Mayou, R.A., Sprigings, D.C. \& Birkhead, J. (2000) Psychological and perceptual factors associated with arrhythmias and benign palpitations. Psychosom. Med., 62, 693-702.

Frieling, T., Enck, P. \& Wienbeck, M. (1989a) Cerebral responses evoked by electrical stimulation of the esophagus in normal subjects. Gastroenterology, 97, 475-478.
Frieling, T., Enck, P. \& Wienbeck, M. (1989b) Cerebral responses evoked by electrical stimulation of rectosigmoid in normal subjects. Dig. Dis. Sci., 34, 202-205.

Gray, M.A., Taggart, P., Sutton, P., Groves, D., Holdright, D.R., Bradbury, D., Brull, D. \& Critchley, H.D. (2007) A cortical potential reflecting cardiac function. Proc. Natl. Acad. Sci. USA, 104, 6818-6823.

Gutterman, D.D. (2009) Silent myocardial ischemia. Circ. J., 73, 785-797.

Hollerbach, S., Bulat, R., May, A., Kamath, M.V., Upton, A.R., Fallen, E.L. \& Tougas, G. (2000) Abnormal cerebral processing of oesophageal stimuli in patients with noncardiac chest pain (NCCP). Neurogastroenterol. Motil., 12, 555-565.

Hollerbach, S., Kamath, M.V., Chen, Y., Fitzpatrick, D., Upton, A.R. \& Tougas, G. (1997a) The magnitude of the central response to esophageal electrical stimulation is intensity dependent. Gastroenterology, 112, 1137-1146.

Hollerbach, S., Kamath, M.V., Fitzpatrick, D., Shine, G., Fallen, E., Upton, A.R. \& Tougas, G. (1997b) The cerebral response to electrical stimuli in the oesophagus is altered by increasing stimulus frequencies. Neurogastroenterol. Motil., 9, 129-139.

Jones, G.E., Leonberger, T.F., Rouse, C.H., Caldwell, J.A. \& Jones, K.R. (1986) Preliminary data exploring the presence of an evoked potential associated with cardiac visceral activity. Psychophysiology, 23, 445.

Kanazawa, M., Fukudo, S., Nomura, T. \& Hongo, M. (2001) Electrophysiological correlates of personality influences in visceral perception. JAMA, 286, 1974-1975.

Kanazawa, M., Nomura, T., Fukudo, S. \& Hongo, M. (2000) Abnormal visceral perception in patients with functional dyspepsia: use of cerebral potentials evoked by electrical stimulation of the oesophagus. Neurogastroenterol. Motil., 12, 87-94.

Kanazawa, M., Hongo, M. \& Fukudo, S. (2011) Visceral hypersensitivity in irritable bowel syndrome. J. Gastroenterol. Hepatol., Suppl 3, 119-121.

Kapa, S. \& Somers, V.K. (2008) Cardiovascular manifestations of autonomic disorders. In: Braunwald's Heart Disease: A Textbook of Cardiovascular Disease, 8th ed., edited by P. Libby, R.O. Bonow, D.L. Mann, D.P. Zipes \& E.B. Braunwald. Saunders, Philadelphia, PA, pp. 2171-2183.

Khalsa, S.S., Rudrauf, D., Feinstein, J.S. \& Tranel, D. (2009) The pathways of interoceptive awareness. Nat. Neurosci., 12, 1494-1496.

Kobayashi, S., Abe, Y., Tashiro, M., Koike, T., Iijima, K., Imatani, A., Ohara, S., Watanabe, S., Fukudo, S. \& Shimosegawa, T. (2010) Brain activity following esophageal acid infusion using positron emission tomography. World J. Gastroenterol., 16, 5481-5489.

Lesser, R.P., Koehle, R. \& Lueders, H. (1979) Effect of stimulus intensity on short latency somatosensory evoked potentials. Electroencephalogr. Clin. Neurophysiol., 47, 377-382.

Malliani, A., Recordati, G. \& Schwartz, P.J. (1973) Nervous activity of afferent cardiac sympathetic fibres with atrial and ventricular endings. J. Physiol., 229, 457-469.

Marron, K., Wharton, J., Sheppard, M.N., Gulbenkian, S., Royston, D., Yacoub, M.H., Anderson, R.H. \& Polak, J.M. (1994) Human endocardial innervation and its relationship to the endothelium: an immunohistochemical, histochemical, and quantitative study. Cardiovasc. Res., 28, 1490-1499.

Mellow, M.H., Simpson, A.G., Watt, L., Schoolmeester, L. \& Haye, O.L. (1983) Esophageal acid perfusion in coronary artery disease. Gastroenterology, 85, 306-312.

Mertz, H., Morgan, V., Tanner, G., Pickens, D., Price, R., Shyr, Y. \& Kessler, R. (2000) Regional cerebral activation in irritable bowel syndrome and control subjects with painful and nonpainful rectal distention. Gastroenterology, 118, 842-848.

Montaya, P., Schandry, R. \& Müller, A. (1993) Heartbeat evoked potentials (HEP): topography and influence of cardiac aware- 
ness and focus of attention. Electroencephalogr. Clin. Neurophysiol., 88, 163-172.

Pollatos, O. \& Schandry, R. (2004) Accuracy of heartbeat perception is reflected in the amplitude of the heartbeat-evoked brain potential. Psychophysiology, 41, 476-482.

Pollatos, O., Kirsch, W. \& Schandry, R. (2005) Brain structures involved in interoceptive awareness and cardioafferent signal processing: a dipole source localization study. Hum. Brain Mapp., 26, 54-64.

Rosen, S.D., Paulesu, E., Nihoyannopoulos, P., Tousoulis, D., Frackowiak, R.S., Frith, C.D., Jones, T. \& Camici, P.G. (1996) Silent ischemia as a central problem: regional brain activation compared in silent and painful myocardial ischemia. Ann. Intern. Med., 124, 939-949.

Sabaté, J.M., Bouhassira, D., Poupardin, C., Wagner, A., Loria, Y. \& Coffin, B. (2008) Sensory signaling effects of tegaserod in patients with irritable bowel syndrome with constipation. Neurogastroenterol. Motil., 20, 134-141.

Sarica, Y., Karacan, I., Thornby, J.I. \& Hirshkowitz, M. (1986) Cerebral responses evoked by stimulation of vesico-urethral junction in man: methodological evaluation of monopolar stimulation. Electroencephalogr. Clin. Neurophysiol., 65, 130-135.

Schandry, R., Sparrer, B. \& Weitkunat, R. (1986) From the heart to the brain: a study of heartbeat contingent scalp potentials. Int. J. Neurosci., 22, 261-275.

Schwedel, J.B. \& Escher, D.J. (1964) Transvenous electrical stimulation of the heart. I. Ann. N. Y. Acad. Sci., 111, 972-980.

Serlie, A.W., Erdman, R.A., Passchier, J., Trijsburg, R.W. \& ten Cate, F.J. (1995) Psychological aspects of non-cardiac chest pain. Psychother. Psychosom., 64, 62-73.

Silverman, D.H., Munakata, J.A., Ennes, H., Mandelkern, M.A., Hoh, C.K. \& Mayer, E.A. (1997) Regional cerebral activity in normal and pathological perception of visceral pain. Gastroenterology, 112, 64-72.

Singh, S., Richter, J.E., Hewson, E.G., Sinclair, J.W. \&
Hackshaw, B.T. (1992) The contribution of gastroesophageal reflux to chest pain in patients with coronary artery disease. Ann. Intern. Med., 117, 824-830.

Sinhamahapatra, P., Saha, S.P., Chowdhury, A., Chakrabarti, S.K., Ghosh, A. \& Maiti, B. (2001) Visceral afferent hypersensitivity in irritable bowel syndrome-evaluation by cerebral evoked potential after rectal stimulation. Am. J. Gastroenterol., 96, 2150-2157.

Sleight, P. \& Widdicombe, J.G. (1965) Action potentials in fibres from receptors in the epicardium and myocardium of the dog's left ventricle. J. Physiol., 181, 235-258.

Suzuki, H., Watanabe, S., Hamaguchi, T., Mine, H., Terui, T., Kanazawa, M., Oohisa, N., Maruyama, M., Yambe, T., Itoh, M. \& Fukudo, S. (2009) Brain activation associated with changes in heart rate, heart rate variability, and plasma catecholamines during rectal distention. Psychosom. Med., 71, 619-626.

Tillisch, K., Mayer, E.A. \& Labus, J.S. (2011) Quantitative metaanalysis identifies brain regions activated during rectal distension in irritable bowel syndrome. Gastroenterology, 140, 91-100.

Van Oudenhove, L., Coen, S.J. \& Aziz, Q. (2007) Functional brain imaging of gastrointestinal sensation in health and disease. World J. Gastroenterol., 13, 3438-3445.

Van Oudenhove, L., Geeraerts, B. \& Tack, J. (2008) Limitations of current paradigms for visceral sensitivity testing. Neurogastroenterol. Motil., 20, 95-98.

Watanabe, S., Hattori, T., Kanazawa, M., Kano, M. \& Fukudo, S. (2007) Role of histaminergic neurons in hypnotic modulation of brain processing of visceral perception. Neurogastroenterol. Motil., 19, 831-838.

Zhang, F.X., Liu, X.J., Gong, L.Q., Yao, J.R., Li, K.C., Li, Z.Y., Lin, L.B., Lu, Y.J., Xiao, H.S., Bao, L., Zhang, X.H. \& Zhang, X. (2010) Inhibition of inflammatory pain by activating B-type natriuretic peptide signal pathway in nociceptive sensory neurons. J. Neurosci., 30, 10927-10938. 